\title{
Research on Construction of Road Network Database Based on Video Retrieval Technology
}

\author{
Fengling Wang $^{1}$ \\ ${ }^{1}$ Hezhou University, School of Mathematics and Computer Hezhou Guangxi 542899, China
}

\begin{abstract}
Based on the characteristics of the video database and the basic structure of the video database and several typical video data models, the segmentation-based multi-level data model is used to describe the landscape information video database, the network database model and the road network management database system. Landscape information management system detailed design and implementation of a detailed preparation.
\end{abstract}

\section{Introduction}

Video database in the field of distance education, telemedicine, e-commerce, digital library, video surveillance and other fields have a wide range of applications. It has become one of the hot spots in multimedia technology research. As the video database technology theory and technology although there are some video database prototype, but in this area there is no complete theoretical system. In many ways, there is no proven way to mature, and there is no widely used commercial video database software platform. It is still in the laboratory research and promotion stage.

\section{Video Database Features}

Video data refers to the storage of audio and video information is a very special data, it is far greater than the transmission of static images and text information, in general, video data and text data has the following characteristics:

\subsection{Video Data has a High Information Resolution.}

The so-called information resolution refers to the number of details provided by the media, such as the description of the crime scene of the video data, we can from crime sites, backgrounds, criminals, criminal tools and criminal means as well as other details.

\subsection{The relationship between video data is complex, is unstructured data organization.}

In the video segment, the time between the video segment on the object relationship, as well as the relationship between the space, in addition, the video data also has a specific application areas.

\subsection{The diversity and ambiguity of video data interpretation.}

It does not have a complete objective explanation of the character numerical data, and there are often personal subjective factors. Because of the ambiguity of video data, when we conduct content-based queries, we can not query specific records as traditional database searches, so we can only query similarity [1].

\section{The Basic Structure of the Video Database}

According to the characteristics of the video database, in order to achieve the insertion and retrieval of video data, the structure of the video database has its own characteristics. However, there is no standard video database architecture, the existing design is mainly for specific applications, the following video database structure to do a simple introduction [2].

\subsection{The hierarchical structure of the video database}

The first layer of the video database is the media support layer, which is the main video segmentation and identifies the original information and other operations. Segmentation means that the video stream is divided into meaningful cells and is identified by identifying the video description information. The second layer is called the data model, which mainly completes the logical 
storage of the physical storage of the video data to the mapping, which is defined in the logical content definition, as well as the data and data relationships determined by the data model. This layer is actually an implementation of the conceptual model of the database. The third layer is the video user interface layer, which completes the video query description and query results on the user information.

\subsection{The basic structure of video database system}

The establishment, use and maintenance of the video database requires the support of the video data management system. Video data management system based on the operating system, unified management and control database, the user through the video data management system to insert and retrieve database commands, such as implementation. At present, the video data management system is not a unified structure, which is due to the current video database management does not exist a complete theoretical system, technically immature, the researchers are still in the exploratory stage, the proposed video data management system is also often for a specific category s application.

The video data management system consists of the following components.

\subsubsection{Video Database}

The video database (VDB) is a physical storage of video data that is often stored on VDB in compressed digital video. Data storage format and system used by the file system. VDB should use an efficient, easy-to-access structure that makes it easy to provide video material.

\subsubsection{Database System}

The video data management system should have a dedicated database system (DBS) that stores a target pattern for video management, which may be used to associate the logical location of the data with the physical location. The structure of the database system and the application of the system and query type support [4].

\subsubsection{Query Processing Unit}

The basic function of the unit is to enable the user to query the video data in a formatted manner. The query formatted by this format can be converted to the target mode in the database system. In the actual video database system, the mode usually describes the video. The attribute's attribute representation matches the existing pattern in the system to achieve the physical location of the query video. The user's query request can be represented using a specially designed video data query language, and sometimes an example of a query can also be used. Obviously, the unit is an important module for the user to retrieve video data.

\subsubsection{Insert the Module}

Insert module is one of the important modules of video data management system. Use this module to insert the original video data into the video database. Due to the large amount of video data, the module can be automatically or semi-automatic insertion, especially to improve efficiency. The main function of the module is to segment the video stream and extract the video from the target model stored in the database system. The target model is implemented from manual extraction to fully automated [5].

\subsubsection{User Interface}

The user interface in the video data management system has an important role in the user interface, so that the user can easily enter the formatted query request, while the query results visual visual expression. The user interface can also be used to complete the interactive video query task.

\section{Typical Video Data Model}

\subsection{Data Model Based on Video Segmentation}

This data model is presented by Dr. Hampapur. By studying the application and generation of different video data, the model can be used as the basis of video data model design. The data model mainly divides the video data through two segmentation criteria: grammar segmentation criteria and semantic segmentation criteria. The video segment is defined by the start frame tb and the end frame te and has $\mathrm{n}$ different characteristics (F1, $\mathrm{F} 2, \ldots \mathrm{Fn})$, and one feature type (W1, W2, .. Wn) for each feature. The feature is based on the principle of content dependency, time expansibility and label requirements [6].

This model introduces elements such as time relationships, images and video features, which can more fully reflect the temporal and spatial relationships and 
characteristics of video data. It has great reference value for video editing and video database, but this model does not give complete video data The presentation and description of the entity and its relationship can only be used as the main representation of the video data model.

\subsection{Data model based on hierarchical identification}

Due to the lack of flexibility in the data segmentation based on video segmentation, Swanberg et al. Proposed hierarchical model. The bottom of the model is a lens, and the content-based lens describes the characteristics of the lens (e.g., image, sound, etc.) taken from the lens itself. Once the appropriate algorithm has been determined, and because of the characteristics from the video itself, it has a more complete representation. Depending on the temporal and spatial relevance of the different shots, the scene can be constructed semantically and multiple scenes with internal relationships are constructed, and the associated sequence forms a composite unit. This pyramid-based video data model has a reference value for the application of data from small to coarse requirements.

\subsection{Time description class model}

An important feature of video data is the twodimensional structure of time and space, the time dimension model [7]. Breiteneder proposed a time-based model in which there are four types of entities: a cinema entity representing the structure of video information composed of multiple tracks; and a channel entity from the media entity. In addition, each channel is derived from the media. In addition, the model allows the information to be grouped into a set of intermediate entities. The entity layer supports channel combining and reuse. The advantage of the timeline model is that the video user provides a very clear and straightforward expression, from the timeline can clearly see the lens partition, play time. The disadvantage of the timeline model is the use of absolute time coordinates, thus increasing the complexity of video editing. This model can not be considered strictly in the form of a complete video data model that can only be viewed vividly in the main form of video data time series and relationships. This model does not introduce advanced abstractions, contains very few semantics, does not take into account the logical structure of video and video between hierarchies, nor does it include the operation of the video operating mechanism, can not share video material and generate new video streams.

\subsection{Algebraic video data model}

Duda presents an algebraic video model that introduces the hierarchical relationship between video segments and video algebra operations and generates new video streams from the original video segment through video algebra operations. The algebraic data model is a nestable hierarchical structure whose basic elements are algebraic video nodes and some operations contained in nodes. The algebraic video node contains the name of the child node, which may be an algebraic video node or an original video segment. The original video segment can be represented by the interval between the original video name and the original video. The algebraic video node also contains descriptive information about its contents and a combination and presentation information describing the playback characteristics of all its child nodes. Algebraic video model is characterized by video synthesis, reuse, organization, search and browse the basic functions. Video algebra can be used to simulate the complex nested logic of video. Video algebra is the core of this model, the contents of the video, the logical structure and the spatial composition of the access; the basic function of the algebraic video model in the algebraic operation based on the model of the level of unity; model node carrying video synthesis description, representation, query and browse Information, and represent the hierarchical and nested structure of the video at the model level [8].

It can be seen from the above characteristics that the algebraic video data model not only includes the segmentation and temporal relationship of video data, but also provides feature information describing the content of the video segment, supports content-based retrieval, and defines the video performance characteristics, which is a more perfect model.

\subsection{Universal video data frame model}

Hjelsvold proposed a general video data frame model, through the use of physical connection diagram to establish a clearer conceptual model. It provides core concepts and modules. Video segmentation is used to define the structure of a video file. Level abstract structure, support scene, sequence and composite unit of multi-level abstraction. Object - oriented technology for model building. Each video object has a unique object identifier. In the model, the concept of video data context is introduced. Through the context, the original video can be synthesized as a new video, and can generate new semantics. The video query algebra is defined in the model, and the video data can be easily manipulated [9].

The starting point of the generic video data frame model is to try to build a generic video data model. VideoSTAR is based on this model of video storage and retrieval experiment system. One of the shortcomings of the model is that the structure is complex and its use is limited. In addition, the model only proposes a framework that is not discussed in terms of encapsulation, inheritance, sharing, and index generation.

\section{Design of Landscape Information Database Model}


Based on the analysis of several typical video data models, the segmented multi-level data model is used to describe the landscape information video database according to the characteristics of video data.

This is because in the video stream there are two inherent abstractions: the entire video stream and a single frame. For most applications, the entire video stream is too rough for the abstraction layer; on the other hand, a single frame is rarely a unit of interest because even a short video has a large number of frames. Naturally presenting other intermediate levels, such as a lens, a scene, a sequence, a composite unit, and the like. Hjelsvold and Midtstraum define the scene as a collection of space and time-related scenes, with the specified meaning of the scene composition sequence, the relevant sequence of the composite unit. Because different users access different levels of video information, we use a segmented multi-level data model to describe the video database. In order to facilitate the realization of the original video stream through the lens split into the lens as the basic unit of the video stream, these basic units stored in the media to form a storage media library. Plus the function and annotation to form the lens library. With the lens library, you can create a video library as needed to form a user view. The video document may be a scene, a sequence, a composite unit, or the like. Its purpose is to achieve the independence of video data, in order to facilitate the sharing of different users of video data and reuse [10]. Figure 3 shows the organization of the video database.

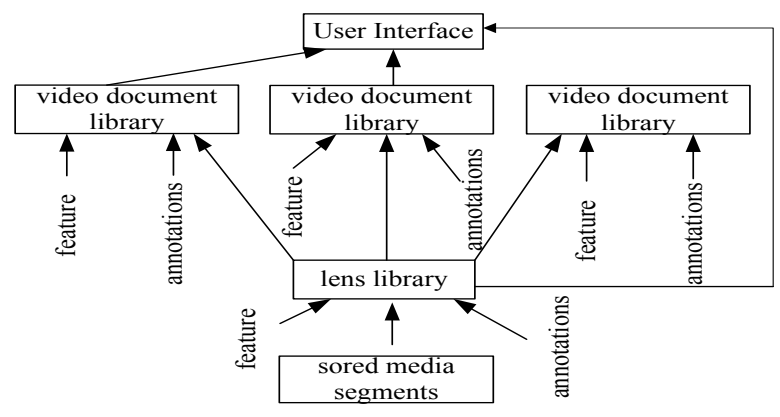

Fig 1. The organization of the video database

The lens library and video library can use the following data model:

$\mathrm{V}$ : video interval: (oid, tb, te)

Features: (F1, F2, ..., Fn)

Note: (A1, A2, ..., An)

Here, oid denotes an interval identifier, and tbte denotes a start frame and a stop frame, respectively. For lenses, this feature is unique to each lens, and the other lenses are not critical. It can be called context-free; also comment on a single shot. For video document libraries, this feature takes into account the relationship between the video intervals, leaving the video interval of the environment, features no longer exist. The note of the video interval depends on the context. This only reflects the time dimension of the video data.

\section{Summary}

Due to the complexity of the video data itself and the wide application, it is still a long way to go to build a common model that can be widely used in many fields. However, as the latest technology for database technology development, content-based retrieval of video database systems is only a beginning, but its prospects are brilliant. With the gradual resolution of the problem, the video database system will play an important role in all areas of the information society.

\section{Acknowledgment}

The author thanks to the support from Hezhou University 2016 Professor of scientific research start fund project under Grant No.HZUJS201615.

\section{References}

1. X.s. Zhou, T. S. Hunag, Exploring the Nature and Variants of Relevance Feedback,in proc. IEEE. Workshop on Content-Based Access of Image and Video Libraries, Hawaii,Dec.2001.

2. Liu w z,Huang J.Multimedia Content Analysis Using Audio and Visual Information.IEEE Signal Processing Magazine,2000,17(6):12-36.

3. Hisashi Miyamori, Shun-ichi lisaku, video annotation for content-based retrieval using human behavior analysis and domain knowledge, Proceedings of the Fourth IEEE International Conference on Automatic Face and Gesture Recognition 2000.

4. $\mathrm{Yu}$ Zhong, Hongji ang Zhang, Anil K. Jain , automatic caption localization in compressed video, IEEE Transactions on Pattern Analysis and Machine Intelligence, Volume 22, Issue 4,April, 2000, pp. 385-392.

5. B. Shahraray, Scene Detection and Content-Based Sampling of Video Sequences, Digital Video Compression: Algorithms and Technologies, Vol. SPIE 2419,February 1995.2-13.

6. Wolf W. Key Frame Selection by Motion Analysis. In: Proc. IEEE Int.Conf. Acoust, Speech, and Signal Proc., 1996

7. Rune Hjelsvold and Roger Roger Midtstrum, Modeling and querying Vedio Data, In Proceedings of the 20th International Conference on Very Large Data Bases, September 1994.

8. Zhu Yingying, Zhou Dongru. A method of extracting key frames from compressed video streams. Computer Engineering and Applications, 2003,18 .

9. Meng Qian. Research on video database data model based on content retrieval. Journal of Xuzhou 
Normal University (Natural Science Edition), 2003,21 (4). 